\title{
Efficient Multi-Objective Synthesis for Microwave Components Based on Computational Intelligence Techniques
}

\author{
Bo Liu \\ Hadi Aliakbarian \\ Soheil Radiom \\ ESAT-MICAS, KU Leuven, Belgium ESAT-TELEMIC, KU Leuven, Belgium ESAT-MICAS, KU Leuven, Belgium \\ Bo.Liu@esat.kuleuven.be \\ Hadi.Aliakbarian@esat.kuleuven.be \\ Soheil.Radiom@esat.kuleuven.be \\ Guy A. E. Vandenbosch \\ Georges Gielen \\ ESAT-TELEMIC, KU Leuven, Belgium \\ ESAT-MICAS, KU Leuven, Belgium \\ Guy.Vandenbosch@esat.kuleuven.be \\ Georges.Gielen@esat.kuleuven.be
}

\begin{abstract}
Multi-objective synthesis for microwave components (e.g. integrated transformer, antenna) is in high demand. Since the embedded electromagnetic (EM) simulations make these tasks very computationally expensive when using traditional multiobjective synthesis methods, efficiency improvement is very important. However, this research is almost blank. In this paper, a new method, called Gaussian Process assisted multi-objective optimization with generation control (GPMOOG), is proposed. GPMOOG uses MOEA/D-DE as the multi-objective optimizer, and a Gaussian Process surrogate model is constructed ON-LINE to predict the results of expensive EM simulations. To avoid false optima for the on-line surrogate model assisted evolutionary computation, a generation control method is used. GPMOOG is demonstrated by a $60 \mathrm{GHz}$ integrated transformer, a $1.6 \mathrm{GHz}$ antenna and mathematical benchmark problems. Experiments show that compared to directly using a multi-objective evolutionary algorithm in combination with an EM simulator, which is the best known method in terms of solution quality, comparable results can be obtained by GPMOOG, but at about 1/3-1/4 of the computational effort.
\end{abstract}

\section{Categories and Subject Descriptors}

\section{2.2. Design Tools and Techniques}

\section{General Terms}

Algorithms, Performance, Design, Theory

\section{Keywords}

Multi-objective microwave components synthesis, Transformer synthesis, Antenna synthesis, Efficient global optimization, MOEA/D, Gaussian Process, Differential evolution

Permission to make digital or hard copies of all or part of this work for personal or classroom use is granted without fee provided that copies are not made or distributed for profit or commercial advantage and that copies bear this notice and the full citation on the first page. To copy otherwise, to republish, to post on servers or to redistribute to lists, requires prior specific permission and/or a fee.

DAC 2012 June 3-7, 2012, San Francisco, California, USA. Copyright 2012 ACM 978-1-4503-1199-1/12/06...\$10.00

\section{INTRODUCTION}

In microwave component design, the designers are typically interested in more than one performance and try to make a tradeoff between them. For instance, for integrated transformer design, RF designers often want to find a tradeoff between efficiency and area. For antenna design, designers are often interested in both the realized gain and the circular polarization axis ratio, as well as other characteristics such as return loss, efficiency, antenna size. Multi-objective optimization is a useful method for these problems. Hence, a growing number of works were reported in recent years focusing on introducing multi-objective optimization algorithms to microwave engineering [1-4], covering microwave filters, antennas, microwave passive components and circuits.

In most of the works, multi-objective evolutionary algorithms (MOEAs) are selected as the search engine, due to their high ability to approximate the Pareto front. Several existing MOEAs based on different evolutionary algorithms (e.g. genetic algorithm, differential evolution, particle swarm optimization) are compared in [1] using the example of a microwave filter; methods based on differential evolution (DE) [5] show the best performance.

Besides the research on investigating better optimizers, a more critical issue is the efficiency improvement needed for many multi-objective microwave component synthesis problems. The computationally cheap equivalent circuit models [6] can only be used when handling low-frequency (a few $\mathrm{GHz}$ ) integrated passive components or very simple antennas, and expensive electromagnetic (EM) simulation is often a must for most microwave component synthesis, especially those working at mmwave frequencies (e.g. $60 \mathrm{GHz}$ ), where parasitic-aware equivalent circuit models are no longer accurate [7]. For single-objective EM simulation-included synthesis, an on-line surrogate model assisted evolutionary algorithm is proposed in [7], which can obtain a highly optimized result in a practical computational time. Compared with existing off-line surrogate model-based global optimization methods [8] and fine-coarse model mapping-based methods [9] solving the same problem, clear advantages on efficiency and optimization ability have been shown.

Although there is a solution for single-objective synthesis problems, multi-objective microwave component synthesis is much more difficult. The goal of multi-objective optimization is 
to generate the Pareto front, which includes a bunch of nondominated points distributed in different areas of the design space, while only one of them is needed for single objective optimization. Even in the computational intelligence field, the available efficient surrogate model assisted multi-objective optimization algorithms can obtain promising results for some benchmark test problems, but not for all of them [10,11]. In the multi-objective microwave component synthesis area, the research on efficiency improvement is still an open research topic. Most of the available works directly use MOEAs as the search engine and EM simulators as the objective function evaluator $[1,2,12]$. Hence, the synthesis or optimization process is typically very CPU time expensive. Efficiency improvement based on hardware resources has been investigated in [2]. Because the evaluation of different candidate designs in a population is independent from each other in most MOEAs, parallel computation is used. However, to the best of our knowledge, there are very few efficient software algorithm for multi-objective EM-simulation-included synthesis.

To address this problem, a new method, called Gaussian Process assisted multi-objective optimization with generation control (GPMOOG), is proposed. The method aims to:

- achieve comparable results as the traditional method (directly using MOEA with an EM simulator) for multi-objective EMsimulation-included synthesis;

- while highly improving the efficiency of the traditional method and making the computational time practical (a couple of hours to about one day).

The remainder of the paper is organized as follows. Section 2 introduces the basic concepts and techniques used in GPMOOG. Section 3 describes the key ideas and algorithm of GPMOOG. Section 4 demonstrates GPMOOG by practical examples and mathematical benchmark problems. The concluding remarks are presented in Section 5.

\section{BASIC CONCEPTS AND TECHNIQUES}

\subsection{Multi-objective optimization and MOEA/D}

Most multi-objective optimization evolutionary algorithms (MOEAs) aim to find a reasonable number of solutions to approximate the Pareto front. A multi-objective optimization problem can be stated as follows [13]:

$$
\min \left\{f_{1}(x), \ldots f_{m}(x)\right\}, x \in \Omega
$$

where $x=\left(x_{1}, \ldots x_{n}\right)$ is the decision variable vector (design variables of the microwave component in this application) and $f_{i}(x)$ are the objective functions, which are the considered desired performances of the microwave component, such as gain, efficiency or area. $\Omega$ is the decision space. A solution $x_{j}$ is said to dominate solution $x_{k}$ if and only if $f_{i}\left(x_{j}\right) \leq f_{i}\left(x_{k}\right)$ for every $i \in\{1, \cdots, m\}$ and $f_{i}\left(x_{j}\right)<f_{i}\left(x_{k}\right)$ for at least one index $i \in\{1, \cdots, m\}$. A point $x^{*} \in \Omega$ is Pareto optimal to (1) if there is no point $x \in \Omega$ such that $f(x)$ dominates $f\left(x^{*}\right) \cdot f\left(x^{*}\right)$ is then a Pareto-optimal objective vector. The set of all the Paretooptimal points is called the Pareto Set (PS). The set of all the Pareto-optimal objective vectors is called the Pareto Front (PF). The PF shows the trade-off curve of different performances.

A state-of-the-art MOEA is the multi-objective evolutionary algorithm based on decomposition (MOEA/D) [14]. It decomposes a multi-objective optimization problem into a set of scalar optimization sub-problems with neighborhood relations. The neighborhood relations are defined by the distances between their aggregation coefficient vectors. In this way, the fitness assignment is the same as single objective optimization, and the diversity is maintained by the diverse search directions determined by the uniformly distributed weight vectors. The first version of MOEA/D used simulated binary crossover and polynomial mutation as the search engines. Later, a new version using the mutation operator (DE/best/1/bin [5]) in $\mathrm{DE}$ as the main search engine was proposed [15] and was shown to outperform MOEA/D and NSGA-II, especially for complex problems. New population updating mechanisms were reported in [15], [16] to improve the diversity of the generated PF. MOEA/D-DE is used as the search engine in the GPMOOG algorithm in this paper. More details of MOEA/D and DE are in [14, 15].

\subsection{Surrogate model assisted evolutionary algorithms}

To boost the efficiency for computationally expensive optimization problems, a promising way is developing surrogate model assisted evolutionary algorithms (SAEAs). The key of SAEA is to employ efficient surrogate models to replace the computationally expensive exact function evaluations (such as EM simulations). As the construction of the surrogate model and its use to predict the function values cost much less effort than directly embedding the expensive exact function evaluator with the optimizer, the computational cost can be reduced significantly. In recent years, many surrogate model construction methods and the corresponding SAEAs have been investigated. Among them, Gaussian Process (GP) or Kriging, artificial neural networks (ANN), support vector machines (SVM) and radial basis function (RBF) show good performances and are widely used [17].

\subsection{Basics of Gaussian Process}

This work uses the Gaussian Process surrogate model, which is introduced briefly in this sub-section. More details are in [18]. GP machine learning not only has very good prediction ability, but also can provide an estimation error with a solid mathematical background. Compared to some other machine learning techniques, the advantages of GP are discussed in section 3.1.

GP predicts a function value $y(x)$ at some design point $x$ by modeling $y(x)$ as a stochastic variable with mean $\mu$ and variance $\sigma$. For two points $x_{i}$ and $x_{j}$, their correlation is defined as:

$\operatorname{Corr}\left(x_{i}, x_{j}\right)=\exp \left(-\sum_{l=1}^{d} \theta_{l}\left|x_{i l}-x_{j l}\right|^{p_{l}}\right), \theta_{l}>0, p_{l} \in[1,2](2)$ where $d$ is the dimension of $x$ and $\theta_{l}$ is the correlation parameter 
which determines how fast the correlation decreases when $x_{i l}$ moves in the $l$ direction. $p_{l}$ is related to the smoothness of the function with $x_{l}$. The optimal values of $\mu, \sigma$ and $\theta$ are determined by maximizing the likelihood function of the observed data. The function value $y\left(x^{*}\right)$ at a new point $x^{*}$ can be predicted as (3):

$$
\begin{aligned}
& \hat{y}\left(x^{*}\right)=\hat{\mu}+r^{T} R^{-1}(y-I \hat{\mu}) \\
& \hat{\mu}=\left(I^{T} R^{-1} I\right)^{-1} I^{T} R^{-1} y \\
& R_{i, j}=\operatorname{Corr}\left(x_{i}, x_{j}\right), i, j=1,2, \cdots n \\
& r=\left[\operatorname{Corr}\left(x^{*}, x_{1}\right), \operatorname{Corr}\left(x^{*}, x_{2}\right), \cdots, \operatorname{Corr}\left(x^{*}, x_{n}\right)\right]^{T}
\end{aligned}
$$

where $x=\left(x_{1}, x_{2} \cdots, x_{n}\right)$ and $y=\left(y_{1}, y_{2} \cdots, y_{n}\right)$ are already evaluated data points and their objective function values. $I$ is a $n \times 1$ vector of ones.

The prediction uncertainty is shown to be:

$$
\begin{aligned}
& \hat{s}^{2}\left(x^{*}\right)=\hat{\sigma}^{2}\left[I-r^{T} R^{-1} r+\left(I-r^{T} R^{-1} r\right)^{2}\left(I^{T} R^{-1} I\right)^{-1}\right] \\
& \hat{\sigma}^{2}=(y-I \hat{\mu})^{T} R^{-1}(y-I \hat{\mu}) n^{-1}
\end{aligned}
$$

In this work, we use the DACE toolbox [19] to implement the Gaussian process-based surrogate model.

\section{THE GPMOOG ALGORITHM 3.1 Key Ideas of GPMOOG}

The surrogate model used in GPMOOG is the GP model. Unlike many other surrogate models, GP does not fit the function to a predefined kernel or structure. The prediction is based on the correlations of the available data. Therefore, GP does not have the problem of over-fitting. In contrast, the over-fitting of the ANNbased surrogate model has been shown in [7].

GPMOOG is an on-line SAEA. The reason why we do not use the off-line SAEA framework is as follows. Off-line SAEA first constructs a good surrogate model which covers the whole design space and then uses it. To obtain a good surrogate model, the training data need to cover the whole design space with a reasonably high density. Hence, a lot of expensive EM simulations are necessary to generate the training data. On the other hand, only a small part of the design space is used in the optimization. The reason is that EA is not based on enumeration, but based on iteration, so many of these expensive EM simulations are wasted. In contrast, in GPMOOG, the surrogate model construction and improvement are performed on-line, so the expensive EM simulations are used only in the necessary area of the design space, which is controlled by MOEA/D-DE. Consequently, GPMOOG is more efficient in terms of the number of EM simulations than methods using an off-line surrogate model.

However, the risk of on-line SAEA is incorrect convergence. Because the surrogate model is constructed based on the available data, which are often not sufficient in the beginning, the corresponding surrogate model may not be reliable. In other words, when using the current surrogate model to predict the newly generated candidate solutions, the prediction values of some points may be far from their true objective function values. In this way, the search may converge to some false optimal points after several iterations [17], and the "good" candidates selected to perform EM simulations to update the surrogate model are also not in the promising area. An intuitive solution to address this problem is to make use of the prediction uncertainty in (4). For newly generated candidates with large prediction uncertainty, EM simulation is used; for those with small prediction uncertainty, the prediction value is used. However, the solution is not trivial. The threshold value for the prediction uncertainty to judge if EM simulation should be used is difficult to decide and is different from problem to problem. Therefore, GPMOOG still makes use of the prediction uncertainty $\hat{s}$ (from (4)) but in a different way.

The GPMOOG method holds two basic ideas. (1) The total number of iterations is equally divided to several groups (e.g. for 100 iterations, we can divide them into 1-10 iterations, 11-20 iterations, ..., 90-100 iterations). In each group, the population in some iterations use expensive EM simulations for candidate evaluation, while for others, prediction values are used. (2) The number of iterations using EM simulation in each group is adaptively adjusted based on the prediction uncertainty of the current surrogate model. Hence, when there are more available samples and the quality of the surrogate model is improved (as reflected by the prediction uncertainty), more iterations use prediction values, which enhances the efficiency considerably. On the other hand, when the quality of the surrogate model is not good enough, more EM simulations are used to maintain correct convergence and to improve the surrogate model. This is the main idea of generation control for the ANN-based single objective SAEA [20]. The rule how to adjust the number of iterations using EM simulation in each group is as follows:

$$
N_{E M}(k+1)=N_{E M(\min )}+\left\lfloor\frac{S(k)}{S_{\max }}\right\rfloor\left(N_{E M(\max )}-N_{E M(\min )}\right)
$$

where $N_{E M(\max )}$ is the maximum number of iterations using EM simulation in a group of iterations. $N_{E M(\min )}$ is the minimum number of iterations using EM simulation in a group. Note that in each group, at least 1 iteration needs to use EM simulation in order to calibrate possible false optima. The $S$ value is the maximum of all prediction uncertainties $\hat{s}$ for the current population using the current surrogate model. Using the maximum $\hat{s}$ value emphasizes the surrogate model quality, which is a safe setting. The search mechanism of GPMOOG is shown in Fig. 1.

\subsection{Handling multiple objectives in GP model}

In MOEA/D-DE, Tchebycheff aggregation is used. The scalar function is as follows:

$$
\operatorname{minimize} g^{t e}\left(x \mid \lambda, z^{*}\right)=\max _{1 \leq i \leq m}\left\{\lambda_{i}\left|f_{i}(x)-z_{i}^{*}\right|\right\}, x \in \Omega
$$

where $\lambda=\left(\lambda_{1}, \cdots, \lambda_{m}\right)$ is a weight vector and $\Sigma_{i=1}^{m} \lambda_{i}=1$, $z^{*}=\left(z_{1}^{*}, \cdots, z_{m}^{*}\right)$ is the reference point, $m$ is the number of objectives. In MOEA/D-DE evolution, different weight vectors will be used for each $f_{i}(x)$ to calculate the corresponding aggregation value. If GP model is directly used to predict 
$g^{t e}\left(x \mid \lambda, z^{*}\right)$, a large number of surrogate models will be generated, because for different $\lambda$ and $z^{*}\left(z^{*}\right.$ is updated in search) the objective function is different. [10] provides an approximation method to predict $g^{t e}\left(x \mid \lambda, z^{*}\right)$ for different weight vectors and reference points by only using the surrogate model for the prediction of $f_{i}(x)$. The derived formula is as follows, more details are in [10]. Consider two objectives $f_{i}(x) \sim N\left(\hat{y}_{i}(x), \hat{s}_{i}^{2}(x)\right)$, and $g^{t e}{ }_{i}(x) \sim N\left(\hat{y}_{i}^{t e}(x),\left(\hat{s}_{i}^{t e}(x)\right)^{2}\right)$.

$\hat{y}_{i}^{t e}(x)=\mu_{1} \Phi(\alpha)+\mu_{2} \Phi(-\alpha)+\tau \phi(\alpha)$

$\left.\hat{s}_{i}^{t e}(x)\right)^{2}=\left(\mu_{1}^{2}+\sigma_{1}^{2}\right) \Phi(\alpha)+\left(\mu_{2}^{2}+\sigma_{2}^{2}\right) \Phi(-\alpha)$

$+\left(\mu_{1}+\mu_{2}\right) \phi(\alpha)-\left(\hat{y}_{i}^{t e}(x)\right)^{2}$

where $\sigma_{i}^{2}=\left[\lambda_{i} \hat{s}_{i}(x)\right]^{2}, \mu_{i}=\lambda_{i}\left(\hat{y}_{i}(x)-z_{i}^{*}\right), i=1,2$.

$\tau=\sqrt{\sigma_{1}^{2}+\sigma_{2}^{2}}, \alpha=\left(\mu_{1}-\mu_{2}\right) / \tau . \phi(\cdot)$ is the standard normal density function, and $\Phi(\cdot)$ is the standard normal distribution function.

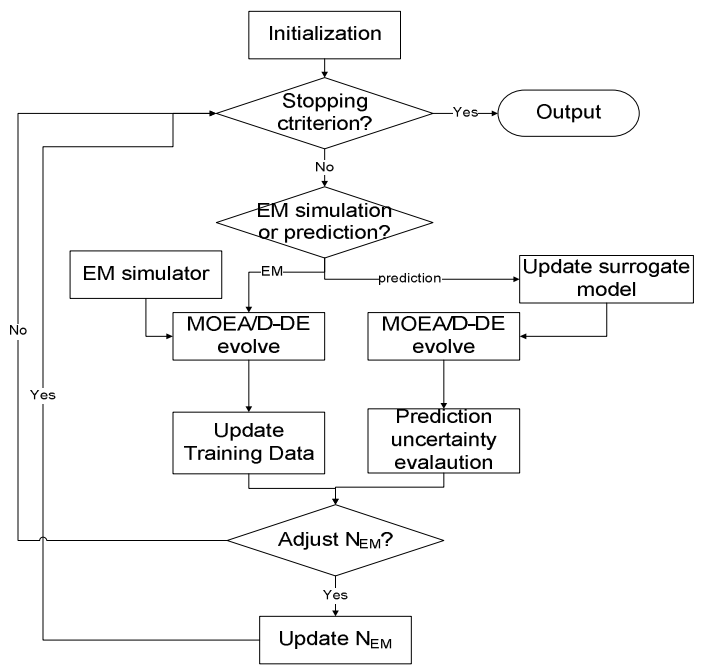

Figure 1. Search mechanism of GPMOOG

\subsection{The GPMOOG algorithm}

The GPMOOG algorithm works as follows.

Input:

(1) a multi-objective EM-simulation-included synthesis problem with $m$ objectives

(2) a stopping criterion (e.g. maximum number of iterations)

(3) MOEA/D parameters: $N$ : the number of sub-problems; $T$ : the neighborhood size; $\delta$ : the probability that parent solutions are selected from the neighborhood; $n_{r}$ : the maximum number of solutions replaced by a child solution; $\lambda$ : weight vector (the generation method is in [14])

(4) evolutionary search parameters: $C R$ : crossover rate in DE, $F$ : the scaling factor in DE (see [5]);

(5) Gaussian Process parameters: a correlation function

(6) Generation control parameters: $C$ : the number of iterations in each group; $N_{E M(\text { init })}$ : the number of iterations using EM simulation in the first group. $N_{E M(\min )}, N_{E M(\max )}$ (see section 3.1)

Output: (1) approximated PF, (2) approximated PS

Procedure:

Step 1: Initialization

Step 1.1: Compute the Euclidean distances between the weight vectors and work out the $T$ closest weight vectors to each weight vector (the set is $B$ ). For $i=1, \cdots N$, set $B(i)=\left\{i_{1}, \cdots, i_{T}\right\} . \lambda^{i_{1}}, \cdots, \lambda^{i_{T}}$ are the $T$ closest vectors to $\lambda^{i}$ (each sub-problem corresponds to a weight vector).

Step 1.2: Randomly generate an initial population $x_{1}, \cdots, x_{n}$. Calculate the fitness values of the population by EM simulations.

Step 1.3: Initialize $z=\left\{z_{1}, \cdots, z_{m}\right\} \quad, \quad$ where $z_{j}=\min _{1 \leq i \leq N} f_{j}\left(x^{i}\right)$.

Step 2: Update

For $i=1, \cdots N$,

Step 2.1: Selection of the mating pool:

Generate a random number which is uniformly distributed in $[0,1]$. Set

$$
P= \begin{cases}B(i) & \text { if rand }<\delta \\ \{1, \cdots, N\} & \text { otherwise }\end{cases}
$$

\section{Step 2.2: Reproduction:}

Set $r_{1}=i$ and randomly select two indexes $r_{2}$ and $r_{3}$ from $P$, and generate a new solution $\bar{y}$ by DE mutation (see [15]). Then, perform a polynomial mutation [14] on $\bar{y}$ to produce a new solution $y$.

\section{Step 2.3: Repair:}

If an element of $y$ is out of the bound of $\Omega$, its value is reset to be a randomly selected value inside the boundary.

Step 2.4: Evaluation of the new candidate (y)

The current iteration is noted as iter.

(2.4.1) If remainder(iter, $C)<N_{E M}$ and remainder(iter, $C) \neq 0$, use EM simulation; otherwise, use GP surrogate model prediction (see equations (3-4)).

(2.4.2) Evaluate the newly generated candidate $y$ by the selected method. When using EM simulation, update the training data set by adding $y$ and the corresponding performances from EM simulation. When using GP surrogate 
model, update the $S_{\max }$ value.

\section{Step 2.5: Update of the reference point:}

For $j=1, \cdots, m$, if $z_{j}>f_{j}(y)$, set $z_{j}=f_{j}(y)$.

\section{Step 2.6: Replacement of solutions:}

(2.6.1) For each $j$ in $\mathrm{P}$, calculate $g\left(y \mid \lambda^{j}, z\right)$ and $g\left(x^{j} \mid \lambda^{j}, z\right)$ by (7) and the $\hat{s}_{i}^{t e}(y)$ value by (8).

(2.6.2) Set $c=0$. If $g\left(y \mid \lambda^{j}, z\right) \leq g\left(x^{j} \mid \lambda^{j}, z\right), \mathrm{c}=\mathrm{c}+1$

(2.6.3) If $c \leq n_{r}, \quad$ for each $j \quad$ with $g\left(y \mid \lambda^{j}, z\right) \leq g\left(x^{j} \mid \lambda^{j}, z\right)$, set $x^{j}=y$. If $c>n_{r}$, for each $j$ with $g\left(y \mid \lambda^{j}, z\right) \leq g\left(x^{j} \mid \lambda^{j}, z\right)$, calculate the Euclidean distances between $f(y)$ and $f\left(x^{j}\right)$ and then rank them. Choose $n_{r}$ solutions with the smallest distances. Set $x^{j}=y$.

\section{Step 2.7: Adjustment of $N_{E M}$ :}

If remainder $($ iter,$C)=0$,

(2.7.1) Using all the $\hat{s}_{i}^{\text {te }}(y)$ from step (2.6.1), calculate the $S$ value of the current surrogate model for the current population by the method described in section 3.1.

(2.7.2) Adjust $N_{E M}$ using (5).

\section{Step 3: Stopping Criterion:}

If the stopping criterion is satisfied, then stop the algorithm and output $\left\{x^{1}, \cdots, x^{N}\right\}$ to Step 4. Otherwise, go to Step 2.

\section{Step 4: Output:}

Perform EM simulation to the last population $\left\{x^{1}, \cdots, x^{N}\right\}$ to obtain their performances $\left\{f\left(x^{1}\right), \cdots, f\left(x^{N}\right)\right\}$. Delete dominated points and output the final PF.

\section{EXPERIMENTAL RESULTS AND COMPARISONS}

In this section, the GPMOOG algorithm is demonstrated for a $60 \mathrm{GHz}$ transformer in a 90nm CMOS technology, a $1.6 \mathrm{GHz}$ antenna in RT5880 technology and mathematical benchmark problems. The parameters are as follows: (1) MOEA/D-DE parameters: $N: 80, T: 8, \delta: 0.9, n_{r}: 1 ; \lambda:$ generated by the method in [14], $C R: 1, F: 0.5$. These are common settings of MOEA/D-DE [15]. (2) Generation control parameters: $C: 5$, $N_{E M(\text { init })}: 4, N_{E M(\min )}: 1, N_{E M(\max )}: 3$. (3) Gaussian Process parameters: the exponential correlation function is used [19]. GPMOOG runs 50 iterations in example 1, and 90 iterations in example 2. These iteration numbers are determined by the improvement of the $\mathrm{PF}$ in each iteration. If no clear improvement is shown in 10 consecutive iterations, GPMOOG is stopped. As the PF is visible for two or three objective multi-objective synthesis, human observation can also be a stopping criterion. The examples are run on a $\mathrm{PC}$ with 4 cores, 12GB RAM and Linux operating system. For the two real-world problems, ADS-Momentum is used as the EM simulator. Because EM simulation is included in example 1 and 2, directly using the traditional method (MOEA/D-DE with the EM simulator without surrogate models) costs a very long time for comparison. Hence, we run the traditional method for example 1 and compare both the generated PF and the efficiency. For example 2, we only analyze the efficiency enhancement since using the traditional method to example 2 will cost a week. Then, the optimization ability comparison is reinforced by mathematical benchmark problems with known PF.

\subsection{Example 1}

The first example is a $60 \mathrm{GHz}$ overlay transformer with octagonal shape in a $90 \mathrm{~nm}$ CMOS process. The design variables are the inner diameter of the primary inductor $(\operatorname{din} p)$, the inner diameter of the secondary inductor (dins), the width of the primary inductor $(w p)$ and the width of the secondary inductor $(w s)$. The ranges of the design variables are dinp, dins $\in[20,150], w p, w s \in[5,10]$ (in $\mu \mathrm{m}$ ). The output impedance is $25 \Omega$. The two objectives are the maximization of the power transfer efficiency (PTE) and the minimization of the square root of the area. The generated PFs of both GPMOOG and the traditional method are shown in Fig.2. Each point in the PF corresponds to a design. An example of the design is shown in Fig. 3.

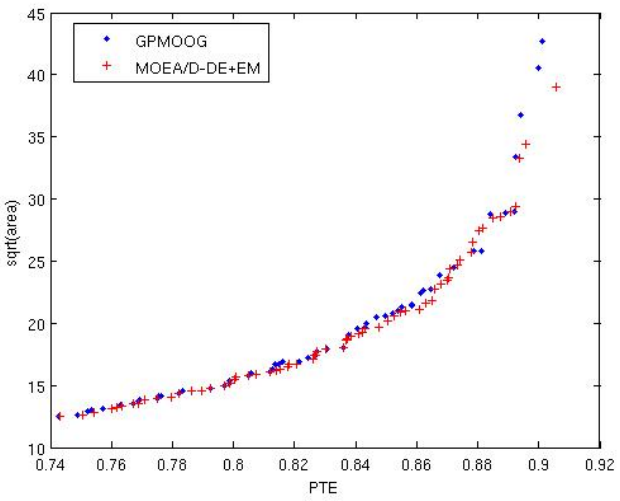

Figure 2. PF generated by GPMOOG and the traditional method (example 1)

From Fig. 2, it can be seen that the PF generated by directly using MOEA/D-DE with the EM simulator is only a slightly better than that of GPMOOG. To quantify the difference, we use:

$\operatorname{err}\left(P F_{\text {GPMOOG }}, P F_{\text {MOEA/D+EM }}\right)-\operatorname{err}\left(P F_{\text {MOEA/D+EM }}, P F_{\text {GPMOOG }}\right)$

where $\operatorname{err}(A, B)$ is defined as: for each point $a$ in $A$ which is dominated by some points in $B$, we calculate $e=\left(\sum_{i=1}^{m}\left|a_{i}-\tilde{b}_{i}\right| /\left|\tilde{b}_{i}\right|\right) / m$, where $m$ is the number of objectives, and $\tilde{b}$ is the nearest nondominated point to $a$ in $B$. err is the average of the $e$ values to all the points. The result is the PF generated by the traditional method is $2.88 \%$ better than GPMOOG. This shows the surrogate model in GPMOOG has good performance. In the first two groups (1-5 iterations, 6-10 iterations), 4 and 3 iterations use EM simulations, respectively. 
After that, the number of iterations that use EM simulation falls to 1 in all the other groups. This shows the adaptive adjustment of $N_{E M}$ and the final results verify the adjustment mechanism. The time consumption of GPMOOG (clock time) is 28.2 hours, which is reasonable for practical use. In the 50 iterations, 16 of them use EM simulation. When directly using MOEA/D-DE with EM simulation, the time consumption is 3.6 days. Hence, a more than 3 times speed enhancement is achieved.
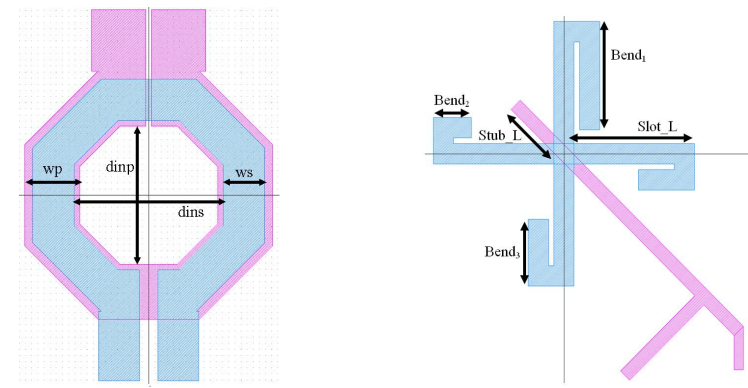

Figure 3. Transformer example Figure 4. Antenna example

\subsection{Example 2}

One of the characteristics often required in antennas is circular polarization (CP). The second example is a miniaturized circular polarized crossed slot antenna fed by using a single feed line based on a work by Vlastis [21]. The antenna has miniaturized crooked arms instead of the Vlastis design, as illustrated in Fig.4. At the same time, because of the unsymmetrical structure, the radiation pattern can rotate and reduce the broadside gain. Therefore, the realized gain and the $\mathrm{CP}$ axial ratio (ARCP) are two important characteristics of the antenna which are taken as the goals of the PF. The antenna is designed at $1.6 \mathrm{GHz}$ frequency. The five main parameters affecting the antenna performances are as follows: lengths of the main arm: Slot L; length of the third part of each arm (in total four arms, one is set to be fixed and the three others are selected as design variables): bend_1, bend_2 and bend 3; the length of the feed stub, Stub L. The ranges of the design variables are: Slot_L $\in[25,35]$, bend_1, bend_2 and bend_3 $3 \in[5,25]$ and Stub_L $\in[5,25]$ (all in $\mathrm{mm}$ ).

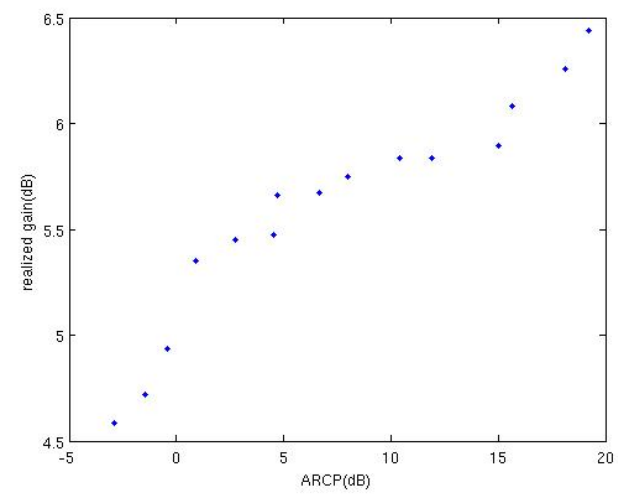

Figure 5. PF showing the trade-off of gain and ARCP (example 2)

The generated PF by GPMOOG is shown in Fig. 5. The result shows the gain versus ARCP tradeoff of the antenna simulated in
ADS-Momentum. Because the ARCP is very sensitive to the five critical parameters, the generated PF is not so smooth. As we would like to have the AR as close as possible to $0 \mathrm{~dB}$, we find out from the PF that the achievable gain range is approximately 5 to $5.5 \mathrm{~dB}$ with small ARCP, which is a very good gain value for a slot antenna having optimized ARCP (slot antenna often has small gain in such size, e.g. 1-2dB).

The synthesis time is 41.5 hours (clock time). In this example, 24 of the total 90 iterations use EM simulation. Considering that the computational cost is dominated by the EM simulation, the speed enhancement is nearly 4 times.

\subsection{Benchmark Tests}

To further verify the optimization ability of GPMOOG, two benchmark problems (ZDT1, ZDT2) [10] for testing MOEAs are used. The number of decision variables is $5 . N$ is set to 100 , and 60 iterations are used. All the other settings are the same as the previous examples. The median results (by IGD value [10]) over 10 runs for both methods are shown in Fig. 6 .
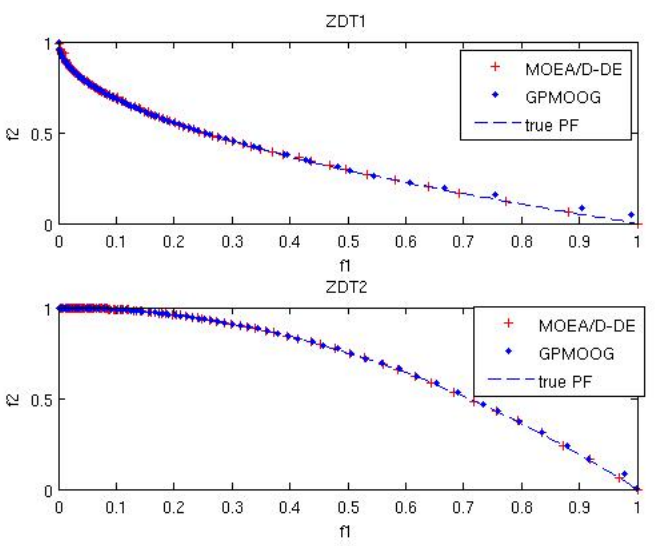

Figure 6. Results of benchmark functions

From Fig. 6, it can be seen that GPMOOG can obtain comparable results as directly using MOEA/D-DE, and the results are also very close to the true PF. In the two examples, 18 of 60 iterations use exact function evaluations. For expensive optimization problems, the efficiency is enhanced for more than 3 times.

\section{CONCLUSIONS}

In this paper, the GPMOOG algorithm has been proposed for the multi-objective synthesis of microwave components. GPMOOG is the first attempt to enhance the efficiency of EM simulationincluded multi-objective synthesis by software algorithms. GPMOOG gets comparable optimization quality and costs 1/3-1/4 of the computational effort as the traditional method, yet. The gain comes from the new surrogate model assisted multi-objective evolutionary algorithm. Future works will focus on expanding the dimensionality of the synthesis problems.

\section{ACKNOWLEDGMENTS}

This research was supported by a special bilateral agreement scholarship of Katholieke Universiteit Leuven, Belgium and Tsinghua University, P. R. China. 


\section{REFERENCES}

[1] Goudos S. K. et al., 2010. Pareto optimal microwave filter design using multiobjective differential evolution. IEEE Transactions on Antennas and Propagation, 132-144.

[2] Poian M. et al., 2008. Multi-objective optimization for antenna design. Proc. of IEEE conference on microwaves, communications, antennas and electronic systems, 1-9.

[3] Oliveria D. et al., 2011. Design of a Microwave Applicator for Water Sterilization Using Multiobjective Optimization and Phase Control Scheme. IEEE Transactions on Magnetic, 1242-1245.

[4] Brito L. C. et al., 2003. A general and robust method for multi-criteria design of microwave oscillators using an evolutionary strategy. Proc. of IEEE international microwave and optoelectronics conference, 135-139.

[5] Price K., et al., 2005. Differential Evolution. A Practical Approach to Global Optimization. Springer, Berlin, Heidelberg, New York.

[6] Nieuwoudt A. et al., 2006. Variability-Aware Multilevel Integrated Spiral Inductor Synthesis. IEEE TCAD, 26132625.

[7] Liu B. et al., 2011. Synthesis of Integrated Passive Components for High-Frequency RF ICs Based on Evolutionary Computation and Machine Learning Techniques. IEEE TCAD, 1458-1468.

[8] Mandal S. K. et al., 2008. ANN- and PSO-Based Synthesis of On-Chip Spiral Inductors for RF ICs. IEEE TCAD, 188192.

[9] Bandler J. et al., 2004. Space Mapping: The State-of-the-Art. IEEE MTT, 337-361.

[10] Zhang Q. et al., 2010. Expensive multiobjective optimization by MOEA/D with Gaussian Process Model. IEEE Transactions on Evolutionary Computation, 456-474.

[11] Knowles J., 2005. ParEGO: A hybrid algorithm with on-line landscape approximation for expensive multiobjective optimization problems. IEEE Transactions on Evolutionary Computation, 50-66.

[12] Chung K. L. et al., 2008. Particle swarm optimization of wideband patch antennas. Proc. of Asia-Pacific microwave conference, 1-4.

[13] Deb K., 2001. Multiobjective optimization using evolutionary algorithms, New York: Wiley.

[14] Zhang Q. et al., 2007. MOEA/D: A multiobjective evolutionary algorithm based on decomposition. IEEE Transactions on Evolutionary Computation, 1-20.

[15] H. Li et al., 2008. Multiobjective optimization problems with complicated Pareto sets, MOEA/D and NSGA-II. IEEE Transactions on Evolutionary Computation, 1-19.

[16] Liu B. et al., 2010. An enhanced moea/d-de and its application to multiobjective analog cell sizing. Proc. of IEEE Congress on Evolutionary Computation, 1-7.

[17] Jin Y., 2005. A comprehensive survey of fitness approximation in evolutionary computation. Soft computing, $3-12$.

[18] Jones D. et al., 1998. Efficient Global Optimization of Expensive Black-Box Functions. J. of Global Optimization, 455-492.

[19] Lophaven S. N. et al., 2002. DACE: A MATLAB Kriging Toolbox, Technical Report. Technical University of Denmark.
[20] Jin Y. et al., 2002. A framework for evolutionary optimization with approximate fitness functions. IEEE Transactions on Evolutionary Computation, 481-494.

[21] Vlasits T. et al., 1996. Performance of a cross-aperture coupled single feed circularly polarised patch antenna. Electronics Letters, 612-613. 